\title{
Response letter to editor: cerebellar mutism and autistic spectrum disorder
}

\author{
Erol Taşdemiroğlu • Miktat Kaya • \\ Can Hakan Yıldırım • Levent Firat
}

Received: 27 December 2010 / Accepted: 28 December 2010/Published online: 1 March 2011

(C) Springer-Verlag 2011

\section{Dear Editor,}

\section{Response:}

We appreciate Dr. Gudrunardottir and colleagues' interests and comments to our recent review.

We gave $5.7-8.5 \%$ incidence rate for cerebellar mutism (CM). In previously and recently published series, $1.5-13 \%$ incidence rate is given [1-4]. Although Catsman-Berrevoets et al. [1] gave $29 \%$ incidence rate for postoperative CM, they did not give any information about the asymptomatic time interval following posterior fossa surgery. Besides the authors did not outline whether postoperative dysarthria and other neurological deficits occurred immediately following posterior fossa surgery or following surgery an asymptomatic brief interval took place. They also stated that they found the highest incidence of postoperative CM. For our opinion, higher incidence rate for $\mathrm{CM}$ in previous series could also be attributed to intraoperative trauma related parenchymal edema, ischemia, and tissue damage. In our opinion, the incidence rates given in our review could be a little lower than the reported series but not close to $29 \%$. As we mentioned in our paper, although $\mathrm{CM}$ is usually described following cerebellar tumor removal, this syndrome could be seen following any posterior fossa surgery. In a recent article of Dubey et al. [2], they reported $1.5 \%$ incidence of $\mathrm{CM}$ among all posterior fossa operations and $8 \%$ in all cerebellar tumor operations.
In our review, we proposed that complete remission from the CM may frequently seen within the 3 months. We did not mean all the CM cases. Recovery period may even exceed to a year.

We only mentioned and discussed the presumed and proposed risk factors and we did not point out any definitive risk factor for the development of postoperative $\mathrm{CM}$. Besides the risk factors, as mentioned in the letter of Dr. Gudrunardottir et al., are still not considered definitive risk factors for development of postoperative CM.

\section{References}

1. Catsman-Berrevoets CE, Van Dongen HR, Mulder PGH, Pas y Geuze D, Paquier PF, Lequin MH (1999) Tumor type and size are high risk factors for the syndrome of cerebellar mutism and subsequent dysarthria. J Neurol Neurosurg Psychiatry 67:755-757

2. Dubey A, Sung W-S, Shaya M, Patwardhan R, Willis B, Smith D, Nanda A (2009) Complications of posterior cranial fossa surgeryan institutional experience of 500 patients. Surg Neurol 72:369-375

3. Pollack IF, Polinko P, Albright AL, Towbin R, Fitz C (1995) Mutism and pseudobulbar symptoms after resection of posterior fossa tumors in children; incidence and pathophysiology. Neurosurg 37:885-893

4. Yildız O, Kabatas S, Yilmaz C, Altınors N, Agaoglu B (2010) Cerebellar mutism syndrome and its relation to cerebellar cognitive and affective function. review of the literature. Ann In Acad Neurol $13: 23-27$

E. Taşdemiroğlu $(\bowtie) \cdot$ M. Kaya $\cdot$ C. H. Yıldırım

Faculty of Medicine, Department of Neurosurgery,

Kafkas University,

Kars, Turkey 36100-TR

e-mail: siberasertas@superonline.com

L. Firat

Neurosurgery Service, Kars State Hospital,

Kars, Turkey 36100-TR 